\title{
Performances zooéconomiques de taurillons de race Borgou complémentés avec du Vitanimal au Bénin
}

\author{
Léopold Sènouwa Guidimê ${ }^{1 *}$ Byll Orou Kpérou Gado ${ }^{2}$ \\ André Jonas Djènontin ${ }^{1}$ Habirou Imorou Sidi ${ }^{3}$ \\ Sévérin Babatoundé ${ }^{3}$
}

\begin{abstract}
Mots-clés
Bovin Borgou, veau, animal à viande, gain de poids, alimentation des ruminants, complément alimentaire pour animaux, Bénin
\end{abstract}

Submitted: 24 April 2020

Accepted: 25 November 2020

Published: 31 March 2021

DOI: $10.19182 /$ remvt.36323

\begin{abstract}
Résumé
Afin de permettre aux animaux d'améliorer leur potentiel de production notamment en saison sèche où le fourrage devient rare et de qualité médiocre, des compléments alimentaires sont de plus en plus mis à leur disposition. L'effet du Vitanimal, aliment fabriqué industriellement, mélange de coques et de tourteau de coton, a été testé chez 15 taurillons de race Borgou à la Ferme d'élevage de l'Okpara afin de mesurer son impact sur la croissance des bovins. Ces derniers ont été répartis en trois lots de cinq taurillons chacun avec un poids moyen de $103 \pm 22 \mathrm{~kg}$. Ils ont reçu la ration de l'aliment testé avant et après le pâturage en parts égales. Le lot 1 sans complément était le lot témoin, les lots 2 et 3 ont bénéficié respectivement de 1 et $2 \mathrm{~kg}$ par jour du complément alimentaire Vitanimal. Les refus ont été quantifiés. Les animaux ont bénéficié également d'eau et de pierres à lécher ad libitum. Des pesées ont été effectuées tous les quinze jours pendant 90 jours. Les analyses ont souligné une différence significative $(p<0,05)$ entre les lots 2 et 3 dans l'ingestion de l'aliment testé. Les résultats ont montré un effet significatif du Vitanimal sur la croissance des taurillons. Les gains moyens quotidiens dans le lot témoin, dans le lot 2 et dans le lot 3 ont été respectivement de $386 \pm 147$ g, 652 \pm 115 g et $927 \pm 160 \mathrm{~g}$. L'impact du Vitanimal a ainsi été fonction de la quantité distribuée et la ration du lot 3 s'est révélée économiquement plus rentable, soit $238 \%$ de taux de rentabilité économique contre $232 \%$ pour le lot 2.

- Comment citer cet article : Guidimê L.S., Kpérou Gado B.O., Djènontin A.J., Imorou Sidi H., Babatoundé S., 2021. Weight-gain and economic performance of Borgu bull calves supplemented with Vitanimal in Benin. Rev. Elev. Med. Vet. Pays Trop., 74 (1): 49-54, doi: 10.19182/remvt.36323
\end{abstract}

\section{INTRODUCTION}

Au Bénin, les productions animales, second pôle d'activité économique du secteur agricole après l'agriculture (Dugué et al., 2003), concernent diverses espèces animales domestiques (bovins, ovins, caprins, porcins, volailles...) mais aussi des espèces non conventionnelles comme l'aulacode (DE, 2016). La viande est de loin la première production animale du pays avec une estimation en 2015 à 23431000 tonnes (CountryStat, 2015). L'élevage bovin, dont dépendent les

\footnotetext{
1. Laboratoire d'écologie, santé et production animales (LESPA), Faculté d'agronomie (FA), Université de Parakou (UP), 01 BP 123, Parakou, Bénin. 2. Ferme d'élevage de l'Okpara (FEO), Direction de l'élevage (DE), Parakou, Bénin.

3. LZ/FSA/UAC, Cotonou, Bénin.

* Auteur pour la correspondance

Tél. : +22966333256 / 67757024 ; email : lguidime@gmail.com
}

viandes produites sur le territoire à hauteur de $57 \%$ (CountryStat, 2015 ; DE, 2016), reste la part importante de la production animale et concerne deux grandes espèces bovines (Bos taurus et Bos indicus). Ces deux espèces seraient issues d'un ancêtre commun originaire probablement du Proche-Orient (Doko, 2014). L'élevage bovin est le plus concentré dans la partie nord-ouest du pays où se trouve la moitié du cheptel estimé à 1202500 têtes (DE, 2006).

Dans ces régions, l'alimentation des bovins est tributaire des parcours naturels et des résidus de récolte (Déhoux et Hounsou-Vê, 1993 ; de Haan, 1997). « La variabilité de la quantité et de la qualité des ressources alimentaires pendant l'année est un facteur limitant très important de la production des bovins » (Archimède et al., 2011 ;Zampaligré, 2012). L'expansion des champs de culture réduit considérablement les aires de pâturage créant un déficit fourrager pour le bétail. Les éleveurs, confrontés aux problèmes de déficit alimentaire s'engagent alors dans des mouvements avec leurs troupeaux à la recherche de ressources fourragères ainsi que dans la mise à la disposition des animaux de compléments alimentaires pour la plupart d'origine artisanale. 
Afin de soutenir l'alimentation du bétail et dans une perspective de sédentarisation des troupeaux face aux conflits entre agriculteurs et éleveurs, l'impact d'un aliment commercial pour bétail nouvellement mis au point (Vitanimal) par l'industrie de transformation agroalimentaire a été évalué sur la croissance de jeunes bovins de race locale Borgou. Le Vitanimal est issu d'un mélange compact de tourteau et de coques de graines de coton. Riche en protéines, hydrates de carbone et matières grasses, c'est un aliment idéal, recommandé pour l'alimentation des ruminants. Produit sous forme de pellets, sa présentation lui confère une manipulation, un stockage et une conservation optimums. Cette expérimentation en station visait à préciser les quantités adéquates à distribuer pour une production optimale de viande bovine.

\section{MATERIEL ET METHODES}

La Ferme d'élevage de l'Okpara, créée en 1952 à l'aide du Fonds d'investissement pour le développement économique et social (FIDES), recouvre 33000 hectares. Elle est située au nord-ouest du Bénin (de $9^{\circ} 06^{\prime}$ à $9^{\circ} 17^{\prime} \mathrm{N}$ ) (figure 1) et bénéficie d'un climat de type soudanien avec une alternance de la saison pluvieuse (juin à septembre) et de la saison sèche (décembre à mars). La pluviométrie annuelle moyenne varie de 858 à $1400 \mathrm{~mm}$ avec environ $1125 \mathrm{~mm}$ enregistrés entre 1999 et 2014 . Les températures varient de 25,3 à $30,5^{\circ} \mathrm{C}$. Le passage d'une saison à l'autre est marqué par une période de transition (avril à mai et octobre à novembre). Les sols rencontrés sont de trois types : les sols lessivés ou non à engorgement de profondeur, occupant la majeure partie de la ferme; les sols hydromorphes riches en argile et en bases échangeables, très fertiles au niveau des bas-fonds et des plaines d'inondation ; et les sols ferrallitiques faiblement dénaturés avec érosion et remaniement, et les sols minéraux bruts. Les principaux groupes socioculturels qui cohabitent dans le domaine sont les Baribas et les Peuls.

\section{Elevage bovin à la Ferme d'élevage de l'Okpara}

La Ferme de l'Okpara est semi-moderne et dotée d'infrastructures (étables, planches, parcs de nuit) pour l'élevage bovin construites en dur. Elle possède un pèse-bétail, un couloir de contention, un pédiluve pour les opérations de déparasitage externes. On y trouve des parcours naturels et de vastes installations fourragères à Panicum maximum, Brachiaria, Aeschynomene histrix et Stylosanthes. En journée (de $9 \mathrm{~h}$ à $17 \mathrm{~h}$ ), les animaux, régulièrement repartis selon les classes d'âges et le sexe, sont conduits au pâturage par des bouviers peuls où ils profitent de leur aliment de base. Cependant, les veaux de moins de quatre mois sont gardés dans des parcs, et ceux de plus de quatre mois et proches du sevrage pâturent à proximité de la ferme. A leur retour, les troupeaux sont enfermés dans leurs abris où ils reçoivent eau et pierres à lécher ad libitum, et accessoirement des compléments alimentaires, notamment les vaches allaitantes et les animaux faibles. Ils s'abreuvent à partir d'un château d'eau construit sur la ferme mais aussi à partir des effluents de la rivière Okpara qui est proche.

La reproduction est principalement basée sur la monte, organisée par un responsable spécialisé en des périodes précises (janvier-février et août-octobre) avec enregistrement des dates de saillie et de mise bas. Les vêlages sont également enregistrés grâce au centre d'insémination annexé à la ferme. Les veaux se voient attribuer à la naissance une fiche de suivi où est inscrit le numéro du parc et de la boucle de la mère, la date de naissance, le sexe, les poids à la naissance et à âge type. Le suivi sanitaire des animaux est assuré par un programme de prophylaxie sanitaire et médicale qui implique le nettoyage régulier des étables, des parcs, des mangeoires, des abreuvoirs et la vaccination des animaux contre diverses pathologies. Un rotoluve et des pédiluves sont également installés respectivement aux entrées de la ferme et des étables.

\section{Troupeau expérimental}

Le Vitanimal a été testé sur de jeunes bovins de race Borgou, race autour de laquelle se font tous les programmes de conservation et d'amélioration à la Ferme de l'Okpara. Les taurillons ainsi utilisés pour conduire des travaux ont été choisis selon leur poids et leur âge ; les sujets en croissance ont été ciblés. Le troupeau expérimental a été entretenu sur les pâturages de la Ferme environ sept heures par jour.

Les bovins ont été répartis en trois lots de cinq taurillons de poids vif homogène (poids moyen de $103 \mathrm{~kg} \pm 22 \mathrm{~kg}$ ). Après une période de quinze jours d'adaptation aux nouvelles conditions, la distribution de l'aliment a été faite deux fois par jour; les animaux recevaient la moitié de la ration très tôt le matin et l'autre moitié le soir au retour du pâturage. Le lot 1 constituait le lot témoin, les lots 2 et 3 ont bénéficié respectivement de 1 et $2 \mathrm{~kg}$ par jour du complément alimentaire Vitanimal. Les refus ont été quantifiés. Les animaux étaient attachés à bonne distance les uns des autres dans la grande étable. Chaque animal était ainsi gardé devant sa mangeoire où il recevait sa ration de complément. Les animaux des lots témoins ont été gardés séparément dans un autre parc. Les animaux ont eu de l'eau et de la pierre

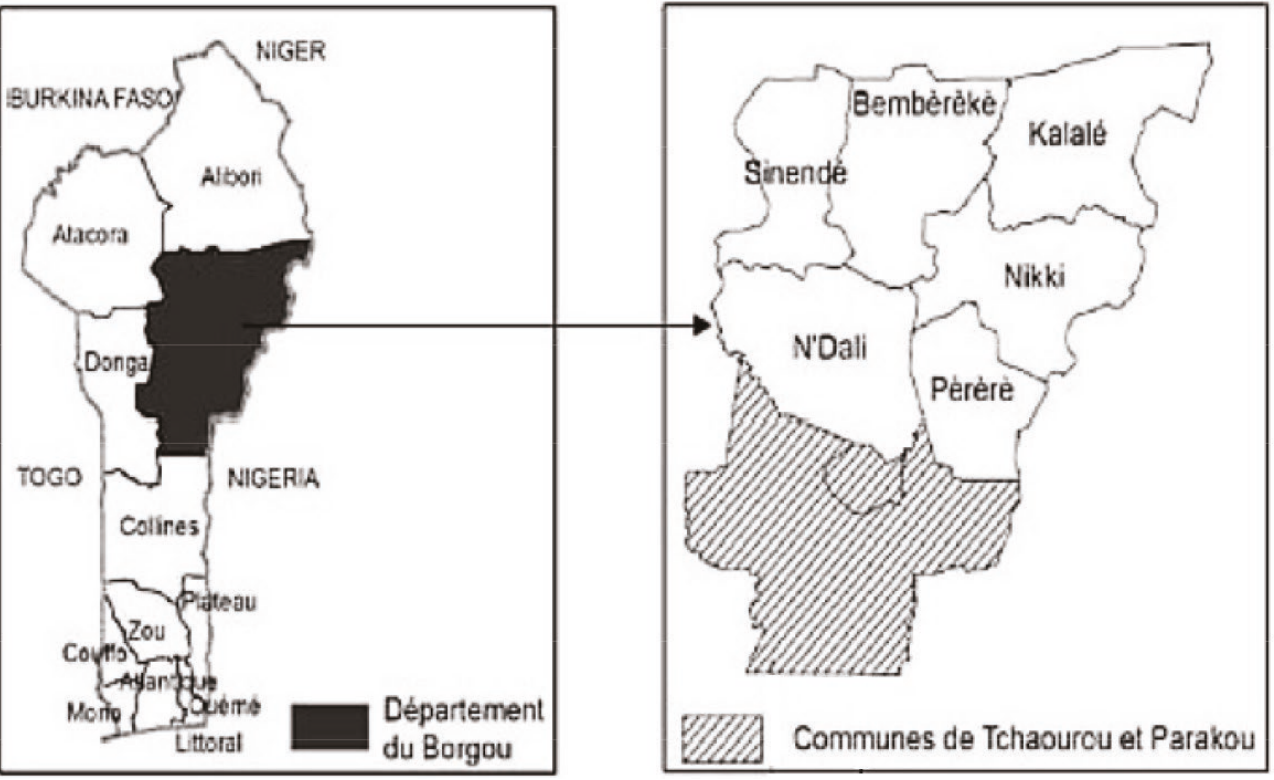

Figure 1 : Localisation de la Ferme d'élevage de I'Okpara au Bénin //I Location of the Okpara Breeding Farm in Benin 
à lécher ad libitum. Des analyses de laboratoire sur la composition chimique et la valeur alimentaire (nutritionnelle) ont été effectuées sur un échantillon du complément alimentaire.

\section{Collecte des données}

Les gains de poids ont été collectés pendant 90 jours. Les numéros d'identification des animaux ont été enregistrés. Ils ont été pesés chaque deux semaines à l'aide d'un pèse-bétail de portée $1000 \mathrm{~kg}$ $\pm 10 \mathrm{~kg}$. Les poids ont été reportés sur des fiches techniques de collecte pour la détermination des gains de poids moyens quotidiens (GMQ). Des coupes sur les aires pâturées par les animaux ont été faites tous les quinze jours afin d'estimer le disponible fourrager dans le temps.

\section{Détermination des paramètres zootechniques}

L'ingestion a été calculée à partir de la formule : Ingestion $(\mathrm{kg} /$ jour) = Quantité d'aliments distribuée (kg/jour) - Refus (kg/jour).

Les performances pondérales mesurées ont été le gain de poids (GP) et le GMQ calculés de la manière suivante : GP $(\mathrm{kg})=$ Poids final (kg) - Poids initial (kg) ; GMQ (g) = Gain de poids (g) / Durée d'embouche (jour).

L'indice de consommation (IC) concernant les performances pondérales a été calculé par la relation : $\mathrm{IC}=$ Quantité d'aliment ingéré ( $\mathrm{g}$ ou kg) / Production (g ou kg).

\section{Indicateurs économiques}

Les indicateurs économiques liés à l'utilisation du Vitanimal ont été déterminés dans une relation d'interdépendance. La performance de l'aliment a été déterminée par la différence entre le GMQ moyen des lots 2 et 3 et celui du lot témoin. Le produit de cette performance avec le prix de vente au kilogramme de poids vif (PV) de l'animal (1000 FCFA $/ \mathrm{kg}$ de PV, soit 1,53€/kg de PV) a permis d'avoir sa valeur économique. La marge nette a été obtenue en faisant la différence entre la valeur économique et le coût de l'aliment.

\section{Analyses statistiques}

Le logiciel Minitab 2016 a été utilisé pour l'analyse des données sur l'ingestion et les performances pondérales. Ces analyses ont porté essentiellement sur les analyses de variance (Anova) à un facteur. Les valeurs moyennes ont été comparées entre elles à l'aide du test de Student Newman Keuls au seuil de $5 \%$. Les probabilités de signification associées aux valeurs de Fisher ont été calculées.

\section{RESULTATS}

\section{Composition chimique}

La composition chimique du complément alimentaire a montré des valeurs élevées de la matière azotée totale $(24,39 \%)$, de la matière organique $(92,06 \%$ ) et de très faibles valeurs de cendres $(7,24 \%)$. La valeur en cellulose brute (35\%) a indiqué sa richesse en fibres, idéale pour le bon fonctionnement du rumen. Les valeurs de la fibre insoluble au détergent neutre (NDF) et de la fibre insoluble au détergent acide (ADF) ont été respectivement de 41 et 38

\section{Digestibilité et valeur alimentaire}

Les valeurs de digestibilité de la matière organique à la cellulase (dMOc), d'énergie et d'azote du Vitanimal ont été bonnes avec une dMOc de 67,61 \%. De même les données énergétiques ont montré que l'aliment était très riche en énergie (unité fourragère viande [UFV] 0,95 et unité fourragère lait [UFL] 1,14). Il a présenté une quantité de matière azotée digestible (MAD) très élevée (191,38 g/kg de matière sèche [MS]). Il en était de même du rapport MAD/UFL indiquant la valeur azotée de l'aliment, dont la valeur $(167,87 \mathrm{~g})$ a été supérieure à celle minimale (130 g) préconisée par Boudet (1991) pour la ration des bovins destinés à l'engraissement. La DMO du Vitanimal était au-dessus de la moyenne, ce qui traduisait son aptitude à être assez rapidement dégradé au niveau du rumen par la flore microbienne et donc à être facilement digéré.

\section{Biomasse des pâturages artificiels à Panicum maximum}

La biomasse fourragère des pâturages à Panicum maximum exploités par le troupeau a été dynamique pendant la période d'expérimentation. Le disponible fourrager a ainsi considérablement baissé tout au long de la saison sèche. Les valeurs de production ont varié de $3900 \mathrm{~kg}$ de MS/ha mi-janvier à $2383 \mathrm{~kg}$ de MS/ha fin février.

\section{Ingestion alimentaire}

L'ingestion volontaire au pâturage des animaux de tous les lots a évolué d'une période à l'autre. Durant les quatre premières semaines de l'étude, le fourrage était disponible et facilement accessible par les animaux des différents lots ; ensuite les pâturages se sont dégradés jusqu'à la fin de l'expérimentation avec l'évolution de la sécheresse. L'ingestion du complément alimentaire par les animaux a varié d'une ration à l'autre. Elle a été de $0,99 \mathrm{~kg}$ et de $1,89 \mathrm{~kg}$ respectivement pour les lots 2 et 3 , avec une différence significative $(p<0,05)$. Ces ingestions ont été proportionnelles aux quantités d'aliments reçues mais l'ingestion a été relativement plus grande au niveau du lot 2. La différence entre les refus quotidiens a aussi été significative $(\mathrm{p}<0,05)$ d'un lot à un autre : les refus ont été plus importants dans le lot $3(0,11 \mathrm{~kg})$ qui recevait une quantité plus importante de complément que dans le lot $2(0,01 \mathrm{~kg})$.

\section{Evolution du poids}

Le poids vif des bovins des trois lots a régulièrement augmenté avec des différences d'un lot à l'autre (figure 2). Le lot témoin a enregistré les gains de poids les plus faibles : le poids moyen est passé de $104 \mathrm{~kg}$ en début d'expérimentation à $132 \mathrm{~kg}$ à huit semaines, puis une légère chute a été constatée avant une croissance en fin d'expérimentation ; le lot 2 a enregistré des poids plus importants régulièrement croissants, de $106 \mathrm{~kg}$ au début à $160 \mathrm{~kg}$ à la fin. Enfin, le lot 3 a présenté la croissance de poids la plus forte, de $98 \mathrm{~kg}$ à $170 \mathrm{~kg}$ après les 90 jours d'expérimentation.

\section{Performances pondérales}

Le gain moyen quotidien des animaux des trois lots a beaucoup varié au cours de l'étude (tableau I). Le GMQ du lot témoin a augmenté de $241 \mathrm{~g} / \mathrm{j}$ à un maximum de $655 \mathrm{~g} / \mathrm{j}$ puis a diminué jusqu'à la fin de l'expérimentation ; la valeur moyenne du GMQ de ce lot sur les trois mois a été de $386 \pm 147 \mathrm{~g} / \mathrm{j}$. Le lot 2, avec un GMQ moyen de $652 \pm 115 \mathrm{~g} / \mathrm{j}$, a présenté une tendance évolutive similaire à celle du lot témoin. Le

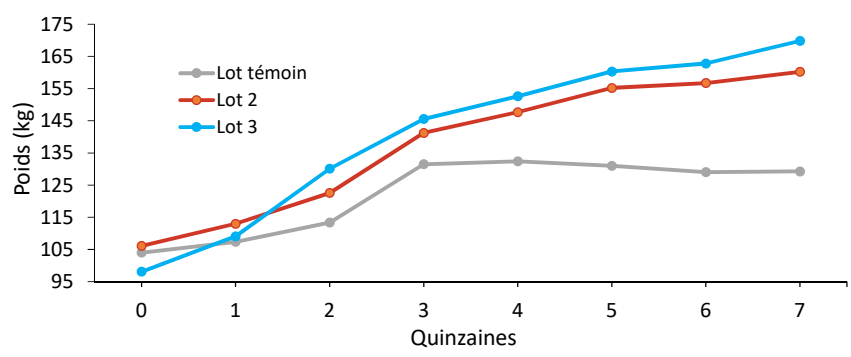

Figure 2 : Evolution du poids des taurillons de race Borgou à la Ferme d'élevage de I'Okpara au Bénin /// Weight growth of Borgu bull calves at the Okpara Breeding Farm in Benin 
Tableau I : Gains de poids moyens quotidiens (GMQ) des taurillons Borgou à la Ferme d'élevage de I'Okpara au Bénin /// Average daily weight gain (GMQ) of Borgu bull calves at the Okpara Breeding Farm in Benin

$\begin{array}{lccc}\text { (g) } & \text { Lot 1 } & \text { Lot 2 } & \text { Lot 3 } \\ \text { GMQ 1 } & 241 \pm 61^{\mathrm{b}} & 493 \pm 85^{\mathrm{ab}} & 786 \pm 98^{\mathrm{a}} \\ \text { GMQ 2 } & 335 \pm 179^{\mathrm{b}} & 588 \pm 132^{\mathrm{b}} & 1143 \pm 431^{\mathrm{a}} \\ \text { GMQ 3 } & 655 \pm 210^{\mathrm{b}} & 836 \pm 74^{\mathrm{ab}} & 1130 \pm 124^{\mathrm{a}} \\ \text { GMQ 4 } & 507 \pm 142^{\mathrm{b}} & 742 \pm 145^{\mathrm{ab}} & 973 \pm 76^{\mathrm{a}} \\ \text { GMQ 5 } & 386 \pm 92^{\mathrm{b}} & 701 \pm 104^{\mathrm{a}} & 889 \pm 72^{\mathrm{a}} \\ \text { GMQ 6 } & 298 \pm 71^{\mathrm{b}} & 602 \pm 85^{\mathrm{a}} & 770 \pm 93^{\mathrm{a}} \\ \text { GMQ 7 } & 281 \pm 107^{\mathrm{b}} & 601 \pm 100^{\mathrm{a}} & 797 \pm 106^{\mathrm{a}}\end{array}$

Lot 1 : témoin non complémenté ; Lots 2 et 3 : complémentés respectivement avec 1 et $2 \mathrm{~kg} /$ jour de Vitanimal ; ${ }^{\mathrm{a}, \mathrm{b}}$ Les valeurs sur une même ligne suivies de lettres différentes sont significativement différentes $(\mathrm{p}<0,05)$ /// Group 1: unsupplemented control, Groups 2 and 3: supplemented with 1 and $2 \mathrm{~kg} /$ day of Vitanimal, respectively; ${ }^{a, b}$ Values on the same line followed by different letters are significantly different $(p<0.05)$

lot 3 a présenté un GMQ d'abord croissant, ensuite plus ou moins constant, avant de diminuer jusqu'à l'arrêt de l'expérimentation ; le GMQ de ce lot sur la période d'observation a été de $927 \pm 160 \mathrm{~g} / \mathrm{j}$.

En dépit d'une tendance croissante des poids notamment au niveau des lots 2 et 3 bénéficiant du complément alimentaire, une baisse du GMQ a été enregistrée dans le temps en raison surtout de la valeur nutritive du fourrage devenue médiocre au pâturage (lignifié, moins de MAT) à cause de la saison sèche. Ces lots supplémentés ont cependant maintenu leurs GMQ au-dessus de ceux du lot témoin. Cette tendance a traduit l'impact réel de l'apport du complément sur le déficit alimentaire.

Les résultats ont ainsi montré des gains de poids en faveur du lot 3. Ces résultats soumis à des tests de comparaison deux à deux de Student ont révélé des différences significatives entre le lot témoin $(\mathrm{p}=0,0025)$ et le lot 2 ( $p<0,05)$, d'une part, et entre le lot $2(\mathrm{p}=0,0022)$ et le lot 3 $(\mathrm{p}<0,05)$, d'autre part, avec une nette différence entre le lot témoin, et les lots 2 et 3 . Les performances pondérales ont ainsi augmenté avec les quantités de Vitanimal distribuées. Cependant, il n'y a pas eu de différence significative $(\mathrm{p}<0,05)$ entre les GMQ des lots 2 et 3 .

\section{Indice de consommation}

La figure 3 montre la représentation de l'évolution de l'indice de consommation du complément alimentaire par les lots 2 et 3 . Les valeurs moyennes des indices de consommation ont été respectivement de 1,56 et 1,96 pour les lots 2 et 3. Les taurillons du lot 2 ont utilisé moins d'aliments que le lot 3 pour produire un kilogramme de viande sur l'ensemble de l'expérimentation. Cette tendance de l'efficacité de l'aliment entre les deux lots en faveur du lot 2 a été beaucoup plus remarquable à partir de la quatrième semaine. La distribution

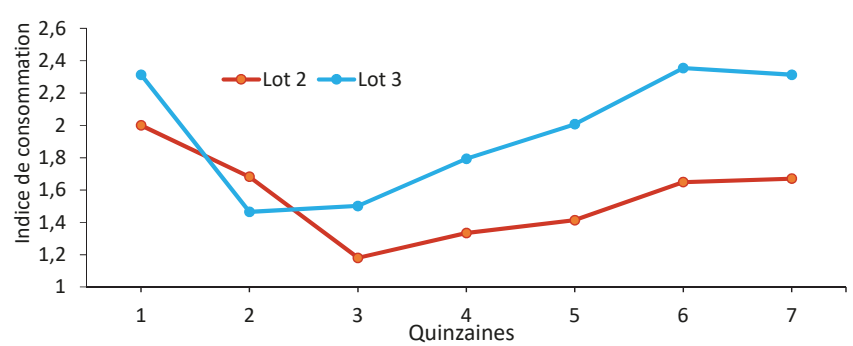

Figure 3 : Evolution de l'indice de consommation du Vitanimal par les taurillons de race Borgou à la Ferme d'élevage de l'Okpara au Bénin /// Trend in the Vitanimal consumption index of Borgu bull calves at the Okpara Breeding Farm in Benin d'un kilogramme par jour du complément aux jeunes bovins en croissance a paru plus rationnelle.

\section{Performances économiques du Vitanimal et marge financière}

L'ingestion du Vitanimal par les taurillons a permis d'obtenir des surplus de GMQ de 266 et $541 \mathrm{~g} / \mathrm{j}$ par taurillon respectivement pour le lot 2 et le lot 3 par rapport au lot témoin (tableau II). Le prix d'un sac de $50 \mathrm{~kg}$ de Vitanimal était de 4000 francs FCA (6,10 euros), soit 80 FCFA $(0,12 €)$ par kilogramme. Sur le marché local, le prix moyen d'un kilogramme de viande était de 1000 FCFA (1,53€). L'analyse des paramètres de rentabilité, notamment la valeur économique de l'animal au poids vif, la marge nette et le taux de rentabilité économique, a montré que les deux lots de taurillons complémentés ont généré des marges financières de $232 \%$ pour le lot 2 et $238 \%$ pour le lot 3 . Ainsi, en investissant $80 \mathrm{FCFA} / \mathrm{kg}$ pour le lot 2 et $160 \mathrm{FCFA}$ pour le lot 3 pour l'acquisition du Vitanimal, l'éleveur a dégagé par jour 186 FCFA $(0,28 €)$ / taurillon pour le lot 2 , et 381 FCFA $(0,58 €)$ / taurillon pour le lot 3 de marge nette. Les marges obtenues ont donc augmenté avec les quantités d'aliments distribuées. A noter que ces fortes marges étaient fonction de la race utilisée pour les travaux, la race Borgou étant une race à viande.

\section{DISCUSSION}

\section{Résultats techniques}

Plusieurs études ont rapporté des quantités ingérées inférieures à celles de 0,99 et $1,89 \mathrm{~kg} / \mathrm{j}$ obtenues avec le Vitanimal lors de cette étude alors que les quantités distribuées à la base étaient supérieures aux nôtres. Le Vitanimal, mélange de coques et de tourteau de coton, s'est avéré plus appété que les compléments utilisés par ces différents auteurs. Le recours aux sous-produits agro-industriels est une solution plus facile à pratiquer, mais leur disponibilité et leur cherté obligent à des arbitrages importants (Magnani, 2020). Ces produits sont utilisés pour complémenter « une ration de base constituée de fourrages. Pour un objectif de production donné, ils sont distribués de façon à équilibrer l'alimentation [au] regard des besoins en énergie et azote de l'animal » (Guerin et al., 2002). Ils augmentent l'ingestion des fourrages en l'occurrence des pailles de $21 \%$ (Sansoucy, 1991).

Les GMQ des lots 2 et 3 ont été supérieurs à ceux de $621 \mathrm{~g} / \mathrm{j}$ rapportés par Houndonougbo et al. (2012) avec une complémentation de la ration des veaux Girolando avec de la graine de coton. Kiéma et al. (2008) rapportent des GMQ de 140 et $162 \mathrm{~g} / \mathrm{j}$ chez des bovins complémentés avec du tourteau de coton à diverses proportions. Ces différences sont imputables non seulement à l'âge des animaux, à la race, à la ration, à la taille des lots expérimentaux mais aussi aux systèmes d'élevage.

Les résultats d'indice de consommation de la présente étude étaient inférieurs à celui de 3,42 rapporté par Houndonougbo et al. (2012) chez des veaux Girolando. Cette différence est due aux différences d'âge et de race des animaux mais aussi au type de complément utilisé. Un indice de consommation encore plus grand $(24,8)$ a été obtenu par Tiémoko et al. (1990) en Côte d'Ivoire en utilisant une ration composée de $75 \%$ de graines de coton chez les bovins Baoulé. Ces différences de valeurs sont imputables non seulement à l'âge, à la race mais aussi au type d'élevage.

\section{Résultats économiques}

L'analyse économique a montré l'effet très avantageux du Vitanimal sur les GMQ qui a permis d'obtenir des gains financiers respectivement de 186 et 381 FCFA $(0,28$ et $0,58 €)$ par kilogramme de viande par jour par taurillon pour les lots 2 et 3, soit $232 \%$ et $238 \%$ de marge par rapport à l'investissement pour l'acquisition du Vitanimal. 
Tableau II : Performances économiques quotidiennes du Vitanimal donné à des taurillons Borgou à la Ferme d'élevage de l'Okpara au Bénin I/I Economic performance of Vitanimal served to Borgu bull calves at the Okpara Breeding Farm in Benin

$\begin{array}{ccccccc}\begin{array}{c}\text { Aliment } \\ \text { distribué (kg) }\end{array} & \begin{array}{c}\text { Coût aliment } \\ \text { (FCFA) }\end{array} & \text { GMQ (g) } & \begin{array}{c}\text { Performance } \\ \text { aliment }(\mathbf{g})^{\mathbf{1}}\end{array} & \begin{array}{c}\text { Valeur économique } \\ \text { de vente PV (FCFA) }\end{array} & \begin{array}{c}\text { Marge nette } \\ (\text { FCFA) })^{\mathbf{3}}\end{array} & \begin{array}{c}\text { Rentabilité } \\ (\%)\end{array} \\ - & - & 386 & - & - & - & - \\ 1 & 80 & 652 & 266 & 266 & 186 & 232 \\ 2 & 160 & 927 & 541 & 541 & 381 & 238\end{array}$

GMQ : gains de poids moyens quotidiens : PV : poids vif ; ${ }^{1}$ Différence entre le GMQ des lots 2 et 3 ayant reçu la complémentation et celui du lot témoin ; ${ }^{2}$ Calculée en multipliant la performance par le prix de vente de PV, soit $1000 \mathrm{FCFA} / \mathrm{kg} \mathrm{de} \mathrm{PV} ;{ }^{3}$ Différence entre la valeur économique et le coût de l'aliment /// GMQ: Average daily weight gain; PV: live weight $;{ }^{I}$ Difference between the GMQ of groups 2 and 3 (supplemented) and that of control; ${ }^{2}$ Calculated by multiplying the performance by the selling price of $P V$, i.e. $1000 \mathrm{FCFA} / \mathrm{kg}$ of $P V ;{ }^{3}$ Difference between the economic value and the feed cost

Montcho et al. (2016) obtiennent des bénéfices par sujet de 1905 FCFA $(2,91 €)$, supérieurs aux nôtres, en incorporant $10 \%$ de tourteau de coton à des blocs multinutritionnels contre $917 \mathrm{FCFA}(1,40 €)$ pour une utilisation de blocs multinutritionnels uniquement; des marges financières supérieures certes mais avec des coûts d'acquisition d'aliment nettement plus élevés (respectivement 3550 et 9050 FCFA, soit 5,42 et $13,81 €$ ) que les nôtres. De même, Alkoiret et al. (2007) rapportent des marges de 4672, 6242 et 5637 FCFA $(7,13,9,53$ et 8,60 €) par sujet après avoir déboursé respectivement 3006, 2136 et 2631 FCFA $(4,59$, 3,26 et $4,02 €$ ) pour l'achat de la ration alimentaire. Ces résultats sont également nettement plus élevés que ceux obtenus dans le cadre de notre étude, aussi bien en marge bénéficiaire qu'en coût alimentaire par sujet. Ces différences de résultats économiques sont imputables non seulement à la nature des sous-produits agro-industriels utilisés, mais aussi à leur disponibilité et leurs prix d'approvisionnement.

\section{CONCLUSION}

La complémentation des bovins dans un contexte de rareté de fourrage en période sèche, de réduction de la mobilité pastorale et aussi dans une opération d'embouche devient nécessaire pour l'amélioration de leur production. Elle améliore les performances du troupeau quand bien même l'approvisionnement en fourrage de base est inadéquat. Cette étude a montré qu'il est possible d'améliorer les performances zootechniques des animaux en les alimentant avec du Vitanimal.
Aucun effet négatif de l'aliment n'a été observé sur les animaux. Il présente une digestibilité satisfaisante, est très riche en énergie et en protéines, peut être assez rapidement dégradé au niveau du rumen et est facilement digéré. Il a notamment eu un impact réel sur les performances pondérales chez des animaux avec des marges financières très satisfaisantes ; les performances zooéconomiques ont augmenté avec les quantités d'aliments distribuées. Ces résultats peuvent susciter un engouement auprès des éleveurs. L'utilisation de cet aliment dans la complémentation des ruminants domestiques améliorerait les performances de production de l'élevage.

\section{Remerciements}

Les auteurs remercient sincèrement la Société des huileries du Bénin à travers le coordonnateur de la recherche, Docteur Adamou MamaSambo, et la Ferme étatique d'élevage de l'Okpara pour avoir respectivement soutenu financièrement et accueilli cette étude.

\section{Déclaration des contributions des auteurs}

AJD et BOKG ont conçu, planifié et supervisé l'étude ; LSG et HIS ont collecté, analysé, interprété les données et rédigé la première version du manuscrit ; SB a révisé le manuscrit.

\section{Conflits d'intérêts}

Les auteurs déclarent qu'ils n'ont aucun conflit d’intérêts.

\section{REFERENCES}

$\overline{\text { Archimède H., Bastianelli D., Boval M., Tran G., Sauvant D., 2011. Ressources }}$ tropicales : disponibilité et valeur alimentaire. Prod. Anim., 24 (1): 23-40, doi: 10.20870/productions-animales.2011.24.1.3235

Boudet G., 1991. Manuel sur les pâturages tropicaux et les cultures fourragères. La documentation française, Paris, France, $266 p$

COUNTRYSTAT/Bénin, 2015. Base de données statistiques. www.fao.org/economic/ess/countrystat/en/ (consulté 18 jan. 2018)

DE, 2006. Afrique de l'Ouest : mobilisation des investissements pour le développement rural et agricole dans la zone CEDEAO, Direction de l'élevage. FAO, Rome, Italie, $53 \mathrm{p}$.

DE, 2016. Rapport annuel 2015. Direction de I'Elevage, République du Bénin, $106 \mathrm{p}$.

De Haan L.J., 1997. Agriculteurs et éleveurs au nord Bénin : écologie et genres de vie. Karthala, Paris, France, 217 p.

Dehoux J. P., Hounsou-Vê G., 1993. Productivité de la race bovine Borgou selon les systèmes d'élevage traditionnels au Nord-Est du Bénin. Rev. Mond. Zootech., 36-48

Doko S.A., 2014. Cours de Zootechnie générale. Faculté d'Agronomie, Université de Parakou, Bénin, 30 p.

Dugué P., Jouve P., 2003. Organisation spatiale et gestion des ressources et des territoires ruraux. Actes du colloque international, 25-27 février 2003, Montpellier, France. Karthala, 217 p.

Guerin H., Lecomte P., Lhoste P., Meyer C., 2002. Généralités sur les ruminants. In: Mémento de l'Agronome. CIRAD, Montpellier, France, 1313-1324
Houndonougbo M.F., Chrysostome C.A.A.M., Babatoundé S., Lokossou H.R., Agbota B., 2012. Fourrages de Moringa oleifera et de Gliricidia sepium utilisés comme compléments alimentaires efficaces pour nourrir des veaux girolando au Bénin. Ann. Sci. Agron., 16 (1): 35-49

Kiéma A., Nianogo A.J., Ouedraogo T., Somda J., 2008. Use of local feed resources in the farmers ram fatting scheme: technical and economic performance. Cah. Agric., 17: 24-27, doi: 10.1684/agr.2008.0154

Magnani S., 2020. Améliorer l'accès des éleveurs à une alimentation du bétail de qualité pour augmenter la production laitière dans les Pays sahéliens d'Afrique de l'Ouest. Note thématique, 20 p.

Montcho M., Babatoundé S., Aboh B. A., Bahini M. J. D., Chrysostome A. A. M. C., Mensah G. A., 2016. Performances zoo-économiques en milieu réel des ovins djallonke complémentés par les blocs multi nutritionnels au Bénin. J. Rech. Sci. Univ. Lomé (Togo), Sér B, 18 (4): 9-22

Sansoucy R., 1991. Problèmes généraux de I'utilisation des sous-produits agro-industriels en alimentation animale dans la région méditerranéenne. In: Tisserand J-L., Fourrages et sous-produits méditerranéens-Zaragoza : CIHEAM. (ed. Alibés X.). Options Méditer. Sér. A, 16: 75-79

Tiémoko Y., Bouchel D., Kouao Brou J., 1990. Growth incidence upon Baoule steers of different levels of cotton seed and molasses supplementation of a fresh and hay diet (Panicum maximum) during their postweaning period Rev. Elev. Med. Vet. Pays Trop., 43 (4): 529-534, doi: 10.19182/remvt.8785

Zampaligré N., 2012. The role of ligneous vegetation for livestock nutrition in the sub-Sahelian and Sudanian zones of West Africa: Potential effects of climate change. PhD thesis, University of Kassel, Germany, 102 p. 


\section{Summary}

Guidimê L.S., Kpérou Gado B.O., Djènontin A.J., Imorou Sidi H., Babatoundé S. Weight-gain and economic performance of Borgu bull calves supplemented with Vitanimal in Benin

In order for animals to improve their production potential, especially during the dry season when fodder becomes scarce and of poor quality, feed supplements are increasingly made available to them. The effect of Vitanimal, an industrially made feed, mixture of husks and cotton cake, was tested on 15 Borgu bull calves at the Okpara Breeding Farm in order to measure its impact on their growth. The cattle were divided into three groups of five bulls each with an average weight of $103 \mathrm{~kg} \pm 22 \mathrm{~kg}$. They were fed the test feed in equal parts before and after grazing. Group 1 was the unsupplemented control, groups 2 and 3 received 1 and $2 \mathrm{~kg}$ per day of Vitanimal feed supplementation, respectively. Refusals were quantified. The animals were also given water and salt blocks ad libitum. Calves were weighed every other week for 90 days. Analyses highlighted a significant difference $(p<0.05)$ between groups 2 and 3 in the tested feed intake. The results showed a significant effect of Vitanimal on the growth of the calves. The average daily gains in the control group, in group 2 and in group 3 were $386 \pm 147 \mathrm{~g}, 652 \pm 115 \mathrm{~g}$ and 927 \pm 160 g, respectively. The impact of Vitanimal depended thus on the amount fed, and the group 3 diet was more cost-effective, with a $238 \%$ return on investment, than group 2 (232\%).

Keywords: Borgu cattle, calves, meat animals, weight gain, ruminant feeding, feed supplements, Benin

\section{Resumen}

Guidimê L.S., Kpérou Gado B.O., Djènontin A.J., Imorou Sidi H., Babatoundé S. Rendimientos zoo económicos de toretes de raza Borgu suplementados con Vitanimal en Benin

Con el fin de permitir a los animales el mejoramiento de su potencial de producción, sobretodo en la estación seca, cuando el forraje escasea y es de calidad mediocre, suplementos alimenticios se ponen con más y más frecuencia a su disposición. Se investigó el efecto de Vitanimal, alimento producido industrialmente, mezcla de conchas y torta de algodón, en 15 toretes de raza Borgu en la Finca de cría de Okpara, con el fin de medir su impacto sobre el crecimiento de los bovinos. Estos últimos se repartieron en tres lotes de cinco toretes cada uno con un peso medio de $103 \mathrm{~kg} \pm 22 \mathrm{~kg}$. Recibieron la ración del alimento investigado antes y después del pastoreo en partes iguales. El lote 1 fue el lote control (sin suplemento), los lotes 2 y 3 beneficiaron respectivamente de 1 y 2 kg por día del suplemento alimenticio Vitanimal. Se cuantificaron los residuos. Los animales también beneficiaron de agua y piedras para lamer ad libitum. Se efectuaron pesajes cada quince días durante 90 días. Los análisis mostraron una diferencia significativa $(p<0,05)$ entre los lotes 2 y 3 para la ingestión del alimento estudiado. Los resultados mostraron un efecto significativo del Vitanimal sobre el crecimiento de los toretes. Las ganancias de peso cotidianas promedio en el grupo control, en el lote 2 y en el lote 3 fueron respectivamente de $386 \pm 147 \mathrm{~g}, 652 \pm 115$ g y $927 \pm 160 \mathrm{~g}$. El impacto del Vitanimal fue resultado en la cantidad distribuida y la ración del lote 3 resultó económicamente más rentable, con una tasa de rentabilidad económica de 238\% contra $232 \%$ para el lote 2.

Palabras clave: ganado bovino Borgu, ternero, animales de carne, ganancia de peso, alimentación de rumiantes, suplementos de piensos, Benín 